\title{
POZİSYON TABANLI ÖĞRENME VE DOĞRUSAL OLMAYAN BOZULMA ETKİSİ ALTINDA İş REDDETMELİ TEK MAKINNE ÇİZELGELEME PROBLEMI
}

\author{
${ }^{1}$ M. Duran TOKSARI, ${ }^{2 B}$ Berrin ATALAY \\ 1,2Erciyes Üniversitesi, Mühendislik Fakültesi, Endüstri Mühendisliği Bölümü, Kayseri, TÜRKIYYE \\ ${ }^{1}$ dtoksari@erciyes.edu.tr, 2berrinucarkus@erciyes.edu.tr
}

(Geliş/Received: 02.04.2018; Kabul/Accepted in Revised Form: 23.05.2019)

\begin{abstract}
ÖZ: Klasik çizelgeleme problemlerinde işlerin işlem sürelerinin sabit olduğu varsayılır ancak gerçek hayat problemlerinde işler öğrenme ve bozulma etkileri altında iken işlem süreleri değişebilmektedir. Ayrıca işlerin işlem sürelerinin fazla olması, üretim ve sabit giderlerin yüksek olması, ekipman ve işgücü yetersizliği gibi sebeplerden dolayı işletmeler ürünlerin işletme içerisinde üretilmemesini isteyebilir. Ürünler dışarıda üretildiğinde bu durum, işletmelere fason maliyeti olarak yansımaktadır. Çalışmamızda, pozisyon tabanlı öğrenme ve doğrusal olmayan bozulma etkisi altında iş reddetmeli tek makineli çizelgeleme problemleri ele alınmıştır. Maksimum tamamlanma zamanı ve reddetme maliyeti minimizasyonu; toplam tamamlanma zamanı ve reddetme maliyeti minimizasyonu olmak üzere iki farklı amaç fonksiyonu ele alınmış, matematiksel programlama ile problemin atama problemi olarak çözülebileceği gösterilmiş, modeller LINGO programı ile çözülmüştür. Bunun yanında maksimum tamamlanma zamanı minimizasyonu probleminin kabul edilen işlerin işlem süresine göre azalan sırada sıralandığında optimum çizelgenin elde edileceği ispatlanmıştır. Bununla birlikte toplam tamamlanma zamanı minimizasyonu probleminin kabul edilen işlerin işlem süresine göre artan sırada sıralandığında optimum çizelgenin elde edileceği ispatlanmıştır. İki farklı öğrenme oranı, iki farklı bozulma oranı ve iki farklı doğrusal olmayan bozulma oranı için ele alınan çizelgeleme problemleri çözülmüş̧ür.
\end{abstract}

Anahtar Kelimeler: İş Reddetme, Doğrusal Olmayan Bozulma Etkisi, Pozisyon Tabanlı Öğrenme, Tek Makine Çizelgeleme Problemi

\section{Under Position Dependent Learning and Nonlinear Deterioration Effect on a Single Machine Scheduling Problem with Job Rejection}

\begin{abstract}
Jobs processing times are constant in classical scheduling problems but in real life problems, jobs processing times can vary because of learning and deterioration effects. Managers may also want to ensure that do not produce products within the companies due to reasons such as excessive processing times, high production costs and fixed costs, equipment and labor shortages. If they want to do jobs outside the companies, they face with outsource cost. In this study, we consider single machine scheduling problem with job rejection under position-dependent learning and nonlinear deterioration effect. There are two scheduling problems with different objective functions. They base on minimization of makespan and minimization of total completion time. It is pointed out that the problem can be solve as assignment problem. For this purpose, a mathematical model is constructed and solved by LINGO. It is proved that for this minimization of makespan objective function, optimum schedule can be obtained when the accepted jobs are scheduled decreasing order according to the accepted jobs' processing times. Furthermore it is proved that for this minimization of total completion time objective function, optimum
\end{abstract}


schedule can be obtained when the accepted jobs are scheduled increasing order according to the accepted jobs' processing times. The scheduling problems under study are solved for two different learning rates, two different deterioration rates and two different nonlinear deterioration rates.

Keywords: Job Rejection, Nonlineer Deterioration Effect, Position-Dependent Learning, Single Machine Scheduling Problem

\section{GİRIŞ (INTRODUCTION)}

Klasik çizelgeleme problemlerinde işlerin işlem sürelerinin sabit olduğu varsayımı vardır ancak gerçek hayat problemlerinde işler öğrenme ve bozulma etkileri altına girebilmekte ve işlerin işlem süreleri değişebilmektedir.

İşçiler zaman içerisinde o işi daha fazla yaptıkları için işi öğrenecek ve işlerin işlem süreleri kısalacaktır. Mosheiov (2001) "öğrenme etkisi" terminolojisini geliştirmiştir. Biskup (1999) öğrenme etkisini çizelgeleme üzerinde kullanan ilk kişidir. Aynı makine özellikleri durumunda, işlerin sayısının fonksiyonuna bağlı olarak işlem zamanının azalacağını varsaymıştır. Gerçek işlem zamanı için aşağıdaki modeli düşünmüştür.

$\mathrm{p}_{\mathrm{j}[\mathrm{r}]}=\left(\mathrm{p}_{\mathrm{j}}\right) \mathrm{r}^{\alpha}$

Burada $p_{j[r]}, r$ pozisyonuna atanan $J$ işinin gerçek işlem zamanı, $p_{j}$ ise temel işlem zamanıdır. $\alpha(\alpha<$ $0)$ öğrenme indeksidir.

Diğer taraftan işlerin işlem sürelerinin fazla olması, üretim ve sabit giderlerin yüksek olması, ekipman ve işgücü yetersizliği gibi sebeplerden dolayı işletmeler ürünlerin işletme içerisinde üretilmemesini isteyebilirler, işlerin fasonda yapılmasını isteyebilirler. Ürünler dışarıda üretildiğinde bu durum, işletmelere fason maliyeti olarak yansımaktadır. İşletmeler, ürünü üretmek isteyip istemedikleri kararını maliyetlere göre değerlendirerek vermektedirler. Direkt üretim maliyetlerinin yanı sıra, reddedilen işler için ceza maliyetleri oluşmaktadır. Bu ceza maliyetleri gelir kaybına sebep olur. Bu durumda çizelgelemeyi yapan kişi hangi işlerin işleneceği, hangi işlerin reddedileceği kararını öncelikli olarak vermek durumunda kalır. Makine çizelgelemede işlerin reddedilmesi durumu ilk olarak Bartal vd (2000) tarafından düşünülmüştür.

Zhang vd (2010) iş reddetme kısıtı altında tek makine çizelgeleme problemi üzerinde çalışmışlardır. Amaçları, kabul edilen işlerin tamamlanma zamanını minimize etmektir. İş ya reddedilir ve reddetme maliyeti ödenir ya da kabul edilir ve tek makinede işlenir. Ancak, reddedilen işlerin toplam reddetme maliyeti verilen üst sınırı aşamayacak şekilde düşünmüşlerdir. Gerstl ve Mosheiov (2012) çalışmalarında pozisyon tabanlı işlem süreleri ve işlerin reddedilmesi birleştirilmiştir. İki amaç fonksiyonu vardır; ilk amaç fonksiyonu, akış zamanı toplamını ve reddedilen işlerin maliyetini minimize etmek, ikinci amaç fonksiyonu ise toplam yüklemeyi ve reddedilen işlerin maliyetini minimize etmektir.

Koulamas ve Panwalkar (2015) tek makine çizelgeleme problemleri için iş reddetme ile erken bitirme ve geç tamamlanma durumları için optimum çözüm veren bir algoritma sunmuşlardır.

Toksarı (2008) tez çalışmasında pozisyon bağımlı öğrenme etkisi ile doğrusal olmayan bozulma etkisi aşağıdaki şekilde tanımlanmıştır.

$p_{[r]}=\left[p_{r}+\left(\alpha \times t_{r}^{b}\right)\right] r^{a}(2)$ 
$p_{[r]}: \mathrm{r}$ pozisyonunda işlenen işin gerçek işlem zamani, $p_{r}: r$ pozisyonunda işlenen işin temel işlem zamanı, $\alpha(\alpha>0)$ bozulma etkisi ve $b(b>0)$ doğrusal olmayan bozulma etkisi, $t_{r}: r$ pozisyonundaki işin başlangıç zamanı ve $a(a<0)$ öğrenme etkisini ifade etmektedir.

Cheng vd (2013)kontrol parametresi öğrenme fonksiyonu olan ve çizelgede, işin gerçek işlem zamanı ile çizelgelenen iş pozisyonunun fonksiyonu olan 2 makineli akış çizelgeleme problemi düşünmüşlerdir. Problemi çözmek için dal sınır algoritması ve üç tavlama algoritması geliştirmişlerdir. Amaçları toplam tamamlama zamanını minimize etmektir.

Liu (2013)elektronik üretim endüstrisinde, elektromanyetik alana maruz kalan parçalardaki kötü etkileri azaltmak için ekstra zamana ihtiyaç duyulduğunu belirtmiş ve bu ekstra zamanı, geçmiş sıra zamanlı teslimat zamanı olarak modellemiştir. Bekleme zamanının oransal değeri şeklinde ifade etmiş, eş paralel makine düşünmüştür. Makalenin üç amacı vardır; işin toplam sapmalarının zamanını minimize etmek, makinelerin toplam yükünü minimize etmek ve toplam tamamlanma zamanını minimize etmektir. Problemin çözümü için polinomiyal algoritma geliştirmiştir.

Azzouz vd(2017) öğrenme etkileri literatürünü incelemiş, yaptıkları çalışmada yapılmış olan çalışmaları sınıflandırmışlardır. İş ya reddedilir ve reddetme maliyeti ödenir ya da kabul edilir ve tek makinede işlenir. Ancak, reddedilen işlerin toplam reddetme maliyetini verilen üst sınırı aşamayacak şekilde düşünmüşlerdir. Toksarı ve Arık (2017)bulanık işlem süreleri ile pozisyon tabanlı bulanık öğrenme etkisi altında tek makine çizelgeleme problemi incelemişlerdir. Bu çalışmada yazarlar maksimum tamamlanma zamanı, toplam tamamlanma zamanı ve toplam ağırlıklandırılmış tamamlanma zamanını minimize etmeyi amaçlamışlardır. Bu üç amaç için bulanık karma tamsayılı doğrusal olmayan programlama modeli kullanmışlardır.

Shabtay (2013) reddetme durumunda zaman tetikleyici işlerin olduğu çizelgeleme problemlerinin genel

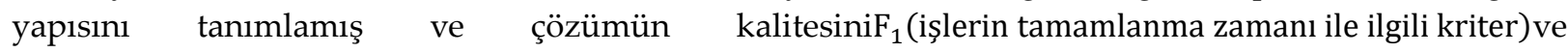
$\mathrm{F}_{2}$ (reddetme maliyeti kriteri) şeklinde ölçmüştür.

Çalışmamızda, pozisyon tabanlı öğrenme ve doğrusal olmayan bozulma etkisi altında iş reddetmeli tek makine çizelgeleme problemi incelenmiştir. İki farklı amaç fonksiyonu çalışılmıştır. Bunlardan ilki maksimum tamamlanma zamanı ve reddetme maliyeti minimizasyonu, diğeri ise toplam tamamlanma zamanı ve reddetme maliyeti minimizasyonudur. Bölüm 2'de maksimum tamamlanma zamanı ve reddetme maliyeti minimizasyonu problemi incelenmiştir ve bir örnek uygulama sunulmuştur. Bölüm 3'te toplam tamamlanma zamanı ve reddetme maliyeti minimizasyonu problemi ele alınmıştır. Bölüm 4'te sayısal sonuçlar karşılaştırılmıştır. Bölüm 5'te sonuçlar verilmiştir.

\section{MAKSIMUM TAMAMLANMA ZAMANI VE REDDETME MALIYETI MINIMIZASYONU(MAXIMUM COMPLETION TIME AND REJECTION COST MINIMIZATION)}

Tek makine ortamından adet işe sahip çizelgeleme problemi düşünülmüştür. $p_{j}: j$ işinin işlem zamanını, $\alpha$ : öğrenme etkisini, $(\gamma>0)$ bozulma etkisini ve $b(b>0)$ doğrusal olmayan bozulma etkisini, $T_{r}: r$ pozisyonundaki işin işe başlama zamanını, $r: 1 \ldots n$ pozisyonu ifade eder. $X_{i j r}$ : karar değişkenidir, $j$ işinin $\mathrm{r}$ pozisyonunda kabul veya red edilme kararıdır. $i=1$, işin kabul edildiğini, $i=2$, işin reddedildiğini ifade eder. $\delta_{j}: j$ işinin ceza maliyetidir. Maksimum tamamlanma zamanı $C_{\max }$ şeklinde ifade edilmiştir.

Aşağıdaki atama problemi çözüldüğünde, maksimum tamamlanma zamanı ve reddetme maliyeti minimizasyonu problemi için optimum sıralama elde edilecektir: 


$$
\begin{aligned}
& \min \sum_{j=1}^{n} \sum_{r=1}^{n} r^{2} \cdot\left(p_{j}+\gamma \cdot\left(T_{r}\right)^{b}\right) r^{\alpha} \cdot X_{1 j r}+\delta_{j} \cdot X_{2 j r} \\
& \sum_{j=1}^{n} X_{i j r} \leq 1 \quad(i=1,2)(r=1,2, \ldots, n)(4) \\
& \sum_{i=1}^{2} \sum_{r=1}^{n} X_{i j r}=1 \quad(j=1,2, \ldots, n) \\
& T_{1}=0 \\
& T_{r}=\sum_{j=1}^{n} \sum_{k}^{r-1}\left[\left(p_{j}+\gamma \cdot\left(T_{k}\right)^{b}\right) \cdot k^{\alpha} \cdot X_{1 j k}\right](r=2,3, \ldots, n) \\
& X_{i j r} \in\{0,1\}
\end{aligned}
$$

Amaç, pozisyon tabanlı öğrenme ve doğrusal olmayan bozulma etkisi altında maksimum tamamlanma zamanı ve reddetme maliyetini minimize etmektir. $r^{2}$ katsayısı işlem zamanını maliyet fonksiyonuna dönüştüren katsayıdır, aynı zamanda kabul edilen işlerin ilk pozisyondan itibaren atanmasını sağlar. (4) numaralı kısıt bir işin herhangi bir pozisyonda kabul ya da reddedilmesi halinde ilgili pozisyonda başka bir işin kabul ya da reddedilmemesini garanti etmektedir. (5) numaralı kısıt, her işin yalnız bir pozisyona kabul veya ret kararlarından biri ile atanmasını garanti etmektedir. (6) numaralı kısit atanan ilk işin işe başlama zamanının 0 olduğunu göstermektedir. (7) numaralı kısıt ilk işten sonraki işlerin işe başlama zamanlarını vermektedir. (8) numaralı kısıt, karar değişkeninin 0 veya 1 değeri almasını sağlamaktadır.

$\mathrm{Bu}$ atama probleminin çözülmesi için $\mathrm{O}\left(n^{3}\right)$ zaman gerekmektedir. Tek makine ortamında maksimum tamamlanma zamanı ve reddetme maliyeti minimizasyonu problemi için aşağıdaki 10 işli örnek çözülmüştür.

\section{Örnek-1}

$\alpha=\log (0.8) / \log (2)=-0,32, \gamma=0.1, b=0.2$ iken, 10 iş için işlem süreleri $[1,25]$ aralığında tam sayılı uniform dağllıma göre üretilmiştir. $\mathrm{p}_{1}=15, \mathrm{p}_{2}=20, \mathrm{p}_{3}=18, \mathrm{p}_{4}=8, \mathrm{p}_{5}=7, \mathrm{p}_{6}=11, \mathrm{p}_{7}=13, \mathrm{p}_{8}=$ $19, \mathrm{p}_{9}=22, \mathrm{p}_{10}=25$. Ceza maliyetleri $[20,60]$ aralığında tam sayılı uniform dağılıma göre üretilmiştir. $\delta_{1}=20, \delta_{2}=30, \delta_{3}=40, \delta_{4}=50, \delta_{5}=45, \delta_{6}=40, \delta_{7}=30, \delta_{8}=60, \delta_{9}=60, \delta_{10}=55$. Problem atama problemi olarak Hungarian metodu ile çözülmüştür. Kabul edilen işlerin optimum sırası $(8,4)$ şeklindedir. $(1,2,3,5,6,7,9,10)$ işleri reddedilmiştir ve optimum amaç değeri 365.176 olarak elde edilmiştir.

Sonuçlar gösteriyor ki, kabul edilen işlerin optimum sırası işlerin işlem sürelerinin azalan sırada sıralanması kuralı (SPT-Shortest Processing Time) ile elde edilmektedir. Aşağıda önerilen teoremin geliştirilmesinde Toksarı ve Güner (2009) tarafından önerilen yaklaşımdan esinlenilmiştir.

Teorem 1: $1\left|r^{2}\left(p_{j}+\gamma\left(T_{r}\right)^{b}\right) r^{\alpha}\right| C_{\max }$ problemi için optimum çözüm işlerin işlem sürelerine göre azalan sirada siralanmasıyla elde edilir. 


\section{İspat:}

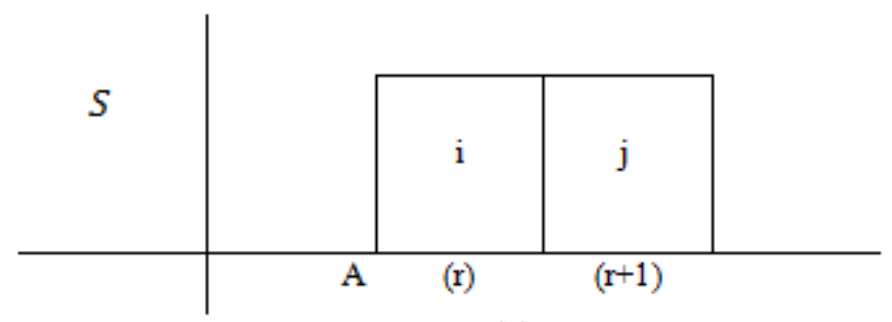

(a)

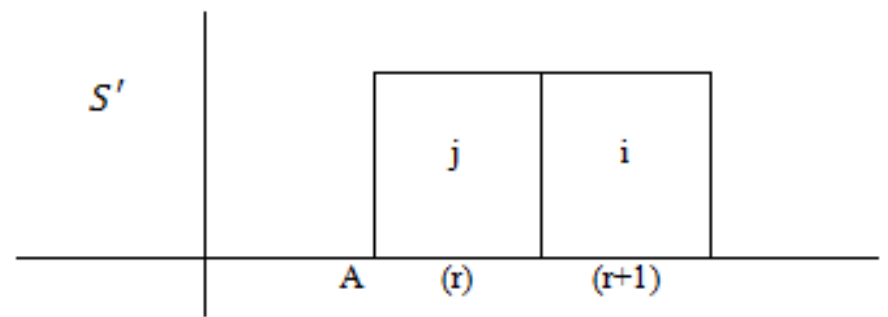

(b)

Şekil 1. i ve j işlerinin $S$ ve $S^{\prime}$ çizelgesinde gösterimi

Figure 1. Display in $S$ and $S^{\prime}$ charts of jobs $i$ and $j$

$p_{i}<p_{j}$ olduğu kabul edilsin.

A: $r$ pozisyonunun öncesinde işlenmiş işlerin toplam tamamlanma zamanı.

Şekil 1. (a)'da gösterilen $S$ çizelgesi için;

$C_{i}(S)=A+r^{2}\left(p_{i}+\gamma T_{r}^{b}\right) r^{\alpha}(9)$

$C_{j}(S)=A+r^{2}\left(p_{i}+\gamma T_{r}^{b}\right) r^{\alpha}+(r+1)^{2}\left(p_{j}+\gamma\left(p_{i}+\gamma T_{r}^{b}\right)^{b}\right)(r+1)^{\alpha}(10)$

Şekil 1. (b)'de gösterilen $S^{\prime}$ çizelgesi için;

$C_{j}\left(S^{\prime}\right)=A+r^{2}\left(p_{j}+\gamma T_{r}^{b}\right) r^{\alpha}$

$C_{i}\left(S^{\prime}\right)=A+r^{2}\left(p_{j}+\gamma T_{r}^{b}\right) r^{\alpha}+(r+1)^{2}\left(p_{i}+\gamma\left(p_{j}+\gamma T_{r}^{b}\right)^{b}\right)(r+1)^{\alpha}$

Eş. (12) ile Eş. (10)'un farklarını aldığımızda;

$$
\begin{aligned}
C_{i}\left(S^{\prime}\right)-C_{j}(S)= & A+r^{2}\left(p_{j}+\gamma T_{r}^{b}\right) r^{\alpha}+(r+1)^{2}\left(p_{i}+\left(p_{j}+\gamma T_{r}^{b}\right)^{b}\right)(r+1)^{\alpha} \\
& -A-r^{2}\left(p_{i}+\gamma T_{r}^{b}\right) r^{\alpha}-(r+1)^{2}\left(p_{j}+\gamma\left(p_{i}+\gamma T_{r}^{b}\right)^{b}\right)(r+1)^{\alpha} \\
C_{i}\left(S^{\prime}\right)-C_{j}(S)= & r^{\alpha+2}\left(p_{j}-p_{i}\right)+(r+1)^{\alpha+2}\left(\left(p_{i}-p_{j}\right)+\gamma\left(p_{j}+\gamma T_{r}^{b}\right)^{b}-\left(p_{i}+\gamma T_{r}^{b}\right)^{b}\right)
\end{aligned}
$$

elde edilir.

$x \geq y>0$ ve $z>0$ iken $\left(y^{z}-x^{z}\right) \leq 0^{\prime}$ dır. Bunun yanında $\alpha<0, p_{i}<p_{j}, b>0$ iken, $C_{i}\left(S^{\prime}\right)-C_{j}(S)<0$ olur.

$\mathrm{S}^{\prime}$ çizelgesi S çizelgesini domine eder. $1\left|r^{2}\left(p_{j}+\gamma\left(T_{r}\right)^{b}\right) r^{\alpha}\right| C_{\text {max }}$ problemi için optimum çizelge işlerin işlem sürelerine göre azalan sırada sıralanmasıyla elde edilir. 


\section{TOPLAM TAMAMLANMA ZAMANI VE REDDETME MALIYETI MINIMIZASYONU (TOTAL COMPLETION TIME AND REJECTION COST MINIMIZATION)}

Tek makine ortamında $n$ adet işe sahip çizelgeleme problemi düşünülmüştür. $p_{j}: j$ işinin işlem zamanını, $\alpha$ : öğrenme etkisini, $(\gamma>0)$ bozulma etkisini ve $b(b>0)$ doğrusal olmayan bozulma etkisini, $T_{r}: r$ pozisyonundaki işin işe başlama zamanını, $r: 1 \ldots n$ pozisyonu ifade eder. $X_{i j r}$ : karar değişkenidir, $j$ işinin r pozisyonunda kabul veya ret edilme kararıdır. $i=1$, işin kabul edildiğini, $i=2$, işin reddedildiğini ifade eder. $\delta_{j}: j$ işinin ceza maliyetidir.

Aşağıdaki atama problemi çözüldüğünde, toplam tamamlanma zamanı ve reddetme maliyeti minimizasyonu problemi için optimum sıralama elde edilecektir:

$\min \sum_{j=1}^{n} \sum_{r=1}^{n} \frac{\mathrm{r}}{2} \cdot(n-j+1) \cdot\left(p_{j}+\gamma \cdot\left(T_{r}\right)^{b}\right) r^{\alpha} \cdot X_{1 j r}+\delta_{j} \cdot X_{2 j r}$

$\sum_{j=1}^{n} X_{i j r} \leq 1 \quad(i=1,2)(r=1,2, \ldots, n)$

$\sum_{i=1}^{2} \sum_{r=1}^{n} X_{i j r}=1 \quad(j=1,2, \ldots, n)$

$T_{1}=0$

$T_{r}=\sum_{j=1}^{n} \sum_{k}^{r-1}\left[\left(p_{j}+\gamma \cdot\left(T_{k}\right)^{b}\right) \cdot k^{\alpha} \cdot X_{1 j k}\right](r=2,3, \ldots, n)(17)$

$X_{i j r} \in(0,1)$

Amaç, pozisyon tabanlı öğrenme ve doğrusal olmayan bozulma etkisi altında toplam tamamlanma zamanı ve reddetme maliyetini minimize etmektir.

$\mathrm{Bu}$ atama probleminin çözülmesi için $\mathrm{O}\left(n^{3}\right)$ zaman gerekmektedir. Tek makine ortamında toplam tamamlanma zamanı ve reddetme maliyeti minimizasyonu problemi için aşağıdaki 10 işli örnek çözülmüştür.

\section{Örnek-2}

$\alpha=\log (0.8) / \log (2)=-0,32, \gamma=0.1, b=0.2$ iken, 10 iş için işlem süreleri $[1,25]$ aralığında tam sayılı uniform dağılıma göre üretilmiştir. $\mathrm{p}_{1}=15, \mathrm{p}_{2}=20, \mathrm{p}_{3}=18, \mathrm{p}_{4}=8, \mathrm{p}_{5}=7, \mathrm{p}_{6}=11, \mathrm{p}_{7}=13, \mathrm{p}_{8}=$ $19, \mathrm{p}_{9}=22, \mathrm{p}_{10}=25$. Ceza maliyetleri $[20,60]$ aralığında tam sayılı uniform dağılıma gore üretilmiştir. 
$\delta_{1}=20, \delta_{2}=30, \delta_{3}=40, \delta_{4}=50, \delta_{5}=45, \delta_{6}=40, \delta_{7}=30, \delta_{8}=60, \delta_{9}=60, \delta_{10}=55$. Problem atama problemi olarak Hungarian metodu ile çözülmüştür. Kabul edilen işlerin optimum sırası $(8,9,10)$ şeklindedir. $(1,2,3,4,5,6,7)$ işleri reddedilmiştir ve optimum amaç değeri 345.5337 olarak elde edilmiştir.

Sonuçlar gösteriyor ki, kabul edilen işlerin optimum sırası işlerin işlem sürelerinin artan sırada sıralanması kuralı (LPT-Longest Processing Time) ile elde edilmektedir. Aşağıda önerilen teoremin geliştirilmesinde Toksarı ve Güner (2009) tarafından önerilen yaklaşımdan esinlenilmiştir.

Teorem 2: $1\left|\frac{r}{2}(n-j+1)\left(p_{j}+\gamma\left(T_{r}\right)^{b}\right) r^{\alpha}\right| \sum C$ problemi için optimum çözüm işlerin işlem sürelerine göre artan sırada sıralanmasıyla elde edilir.

\section{İspat:}

$p_{i}<p_{j}$ olduğu kabul edilsin.

A: $r$ pozisyonunun öncesinde işlenmiş işlerin toplam tamamlanma zamanı.

Şekil 1. (a)'da gösterilen $S$ çizelgesi için;

$$
\begin{aligned}
& C_{i}(S)=A+\frac{r}{2}(n-j+1)\left(p_{i}+\gamma T_{r}^{b}\right) r^{\alpha}(19) \\
& C_{j}(S)=A+\frac{r}{2}(n-j+1)\left(p_{i}+\gamma T_{r}^{b}\right) r^{\alpha}+\frac{r+1}{2}(n-j+1)\left(p_{j}+\gamma\left(p_{i}+\gamma T_{r}^{b}\right)^{b}\right)(r+1)^{\alpha}(20) \\
& \sum C(S)=2 A+r(n-j+1)\left(p_{i}+\gamma T_{r}^{b}\right) r^{\alpha}+(r+1)(n-j+1)\left(p_{j}+\gamma\left(p_{i}+\gamma T_{r}^{b}\right)^{b}\right)(r+1)^{\alpha}(21)
\end{aligned}
$$

Şekil 1. (b)'de gösterilen $S^{\prime}$ çizelgesi için;

$$
\begin{aligned}
& C_{j}\left(S^{\prime}\right)=A+\frac{r}{2}(n-j+1)\left(p_{j}+\gamma T_{r}^{b}\right) r^{\alpha} \\
& C_{i}\left(S^{\prime}\right)=A+\frac{r}{2}(n-j+1)\left(p_{j}+\gamma T_{r}^{b}\right) r^{\alpha}+\frac{r+1}{2}(n-j+1)\left(p_{i}+\gamma\left(p_{j}+\gamma T_{r}^{b}\right)^{b}\right)(r+1)^{\alpha} \\
& \sum C\left(S^{\prime}\right)=2 A+r(n-j+1)\left(p_{j}+\gamma T_{r}^{b}\right) r^{\alpha}+(r+1)(n-j+1)\left(p_{i}+\gamma\left(p_{j}+\gamma T_{r}^{b}\right)^{b}\right)(r+1)^{\alpha}
\end{aligned}
$$

Eş. (24) ile Eş. (21)'in farklarını aldığımızda;

$$
\begin{aligned}
\sum C\left(S^{\prime}\right)-\sum C(S) & =2 A+r(n-j+1)\left(p_{j}+\gamma T_{r}^{b}\right) r^{\alpha}+(r+1)(n-j+1)\left(p_{i}+\gamma\left(p_{j}+\gamma T_{r}^{b}\right)^{b}\right)(r+1)^{\alpha} \\
& -2 A-r(n-j+1)\left(p_{i}+\gamma T_{r}^{b}\right) r^{\alpha}-(r+1)(n-j+1)\left(p_{j}+\gamma\left(p_{i}+\gamma T_{r}^{b}\right)^{b}\right)(r+1)^{\alpha} \\
\sum C\left(S^{\prime}\right)-\sum C(S) & =(n-j+1)\left(p_{i}-p_{j}\right)\left(r^{\alpha+1}+(r+1)^{\alpha+1} \gamma\left(\left(p_{i}+\gamma T_{r}^{b}\right)^{b}-\left(p_{j}+\gamma T_{r}^{b}\right)^{b}\right)\right)
\end{aligned}
$$

elde edilir.

$x \geq y>0$ ve $z>0$ iken $\left(y^{z}-x^{z}\right) \leq 0^{\prime}$ dır.Ayrıca $\alpha<0, p_{i}<p_{j}, b>0$ iken, $\sum C\left(S^{\prime}\right)-\sum C(S)>0$ olur.

S çizelgesi S' çizelgesini domine eder. $1\left|\frac{r}{2}(n-j+1)\left(p_{j}+\gamma\left(T_{r}\right)^{b}\right) r^{\alpha}\right| \sum C$ problemi için optimum çizelge işlerin işlem sürelerine göre artan sırada sıralanmasıyla elde edilir. $\square$ 


\section{SONUÇLAR VE TARTIŞMALAR (RESULTS AND DISCUSSIONS)}

Geliştirilen modeller iki farklı öğrenme oranı (\%80, \%70), iki farklı bozulma oranı $(0.1,0.2)$ ve iki farklı doğrusal olmayan bozulma oranı $(0.2,0.5)$ için model çözülmüştür. Çözümler Çizelge 1 'de verilmiştir.

Çizelge 1. Farklı öğrenme oranı, farklı bozulma oranı ve farklı doğrusal olmayan bozulma oranları için karşılaştırma tablosu

Table 1. Comparison chart for different learning rate, different deterioration rate and different nonlineer deterioration rate

\begin{tabular}{|c|c|c|c|c|}
\hline $\begin{array}{c}\text { Amaç } \\
\text { Fonksiyonu }\end{array}$ & Bozulma Oranı $(\gamma)$ & $\begin{array}{c}\text { Doğrusal Olmayan Boz. } \\
\text { Oram (b) }\end{array}$ & $\begin{array}{c}\text { Öğrenme Oranı } \\
(\alpha)\end{array}$ & Model Sonucu \\
\hline \multirow{8}{*}{$\begin{array}{c}\text { Maksimum } \\
\text { Tamamlanma } \\
\text { Zamanı }\end{array}$} & \multirow{4}{*}{0.1} & \multirow{2}{*}{0.2} & $80 \%$ & 365,176 \\
\hline & & & $70 \%$ & 353,671 \\
\hline & & \multirow{2}{*}{0.5} & $80 \%$ & 365.995 \\
\hline & & & $70 \%$ & 355.968 \\
\hline & \multirow{4}{*}{0.2} & \multirow{2}{*}{0.2} & $80 \%$ & 365,753 \\
\hline & & & $70 \%$ & 355,149 \\
\hline & & \multirow{2}{*}{0.5} & $80 \%$ & 367.210 \\
\hline & & & $70 \%$ & 359.771 \\
\hline \multirow{8}{*}{$\begin{array}{c}\text { Toplam } \\
\text { Tamamlanma } \\
\text { Zaman1 }\end{array}$} & \multirow{4}{*}{0.1} & \multirow{2}{*}{0.2} & $80 \%$ & 345,534 \\
\hline & & & $70 \%$ & 331,090 \\
\hline & & \multirow{2}{*}{0.5} & $80 \%$ & 346.366 \\
\hline & & & $70 \%$ & 333.165 \\
\hline & \multirow{4}{*}{0.2} & \multirow{2}{*}{0.2} & $80 \%$ & 346,039 \\
\hline & & & $70 \%$ & 332,461 \\
\hline & & \multirow{2}{*}{0.5} & $80 \%$ & 347.710 \\
\hline & & & $70 \%$ & 336.451 \\
\hline
\end{tabular}

\section{SONUÇLAR (CONCLUSION)}

Çalışmamızda, pozisyon tabanlı öğrenme ve doğrusal olmayan bozulma etkisi altında iş reddetmeli tek makineli çizelgeleme problemleri ele alınmıştır. Maksimum tamamlanma zamanı ve reddetme maliyeti minimizasyonu ve toplam tamamlanma zamanı ve reddetme maliyeti minimizasyonu olmak üzere iki farklı amaç fonksiyonu geliştirilmiş, matematiksel modeller önerilerek problemin atama problemi olarak çözülebileceği gösterilmiştir. Ayrıca maksimum tamamlanma zamanı minimizasyonu probleminin, işlerin işlem süresine göre azalan sırada sıralanması ile optimum çizelgenin elde edildiği gösterilmiştir. Toplam tamamlanma zamanı minimizasyonu probleminin, işlerin işlem süresine göre artan sırada sıralanmasıyla optimum çizelgenin elde edileceği sunulmuştur.

\section{KAYNAKLAR (REFERENCES)}

Azzouz, A., Ennigrou, M., Ben Said, L., 2017, “Scheduling Problems Under Learning Effects: Classification And Cartography", International Journal of Production Research, 1-20. 
Bartal, Y., Leonardi, S., Marchetti-Spaccamela, A., Sgall, J., Stougie, L., 2000, “Multiprocessor Scheduling with Rejection", SIAM Journal on Discrete Mathematics, 13(1), 64-78.

Biskup, D., 1999, "Single-Machine Scheduling with Learning Considerations", European Journal of Operational Research, 115(1), 173-178.

Cheng, T. C. E., Wu, C. C., Chen, J. C., Wu, W. H., Cheng, S. R., 2013, “Two-Machine Flowshop Scheduling With A Truncated Learning Function To Minimize The Makespan", International Journal of Production Economics, 141(1), 79-86.

Gerstl, E., Mosheiov, G., 2012, "Scheduling On Parallel Identical Machines with Job-Rejection And Position-Dependent Processing Times", Information Processing Letters, 112(19), 743-747.

Koulamas, C., Panwalkar, S. S., 2015, “On the Equivalence of Single Machine Earliness/Tardiness Problems with Job Rejection", Computers \& Industrial Engineering, 87, 1-3.

Liu, M., 2013, "Parallel-Machine Scheduling With Past-Sequence-Dependent Delivery Times And Learning Effect", Applied Mathematical Modelling, 37(23), 9630-9633.

Mosheiov, G., 2001,"Scheduling Problems with a Learning Effect”, European Journal of Operational Research, 132 (2001), 687-693.

Shabtay, D., Gaspar, N., Kaspi, M., 2013, "A Survey On Offline Scheduling With Rejection", Journal of Scheduling, 16(1), 3-28.

Toksarı, M. D., 2008, Öğrenme Ve Bozulma Etkileri Altında Hazırlık Zamanlı Paralel Makineli Erken Tamamlanma/Gecikme Çizelgeleme Problemi, Doktora Tezi, Gazi Üniversitesi, Fen Bilimleri Enstitüsü, Ankara.

Toksarı, M. D., Güner, E., 2009,“Scheduling Problems With The Nonlinear Effects Of Learning And Deterioration", The International Journal of Advanced Manufacturing Technology, 45(7-8), 801-807.

Toksarı, M. D., Arık, O. A., 2017, “Single Machine Scheduling Problems Under Position-Dependent Fuzzy Learning Effect With Fuzzy Processing Times", Journal of Manufacturing Systems, 45, 159-179.

Zhang, L., Lu, L., Yuan, J., 2010,“Single-Machine Scheduling Under the Job Rejection Constraint", Theoretical Computer Science, 411(16), 1877-1882. 